\title{
LA CONVENTION EUROPÉENNE DES DROITS DE L'HOMME ET LES CONFLITS DE LOIS : SYNTHĖSE DE DIX ANS DE JURISPRUDENCE EUROPÉENNE
}

\author{
PATRICK KINSCH*
}

\begin{abstract}
The European Convention on Human Rights and the conflict of laws: summary of ten years of European case-law

The European Court of Human Rights, whose jurisprudence is presented here, is not a court specialising in private international law. However it applies the norms deriving from the European Convention on Human Rights to applications by litigants complaining of any type of national legal rules, including the rules of private international law. There are cases decided by the European Court in all fields of private international law, out of which the cases relating to the field of choice of law are presented here. The cases decided during the last ten years can be classified in cases on the human rights control of (1) equality before the national rules of choice of law, (2) the public policy exception, (3) fraus legis, and (4) the methodological choice between the application, to a status acquired abroad, of the forum's own rules of choice of law, or, alternatively, the method of recognition of the result of the application of the foreign rules of choice of law. The case-law of the European Court has had, on the whole, a moderate - and moderating - effect on national choice of law rules.
\end{abstract}

Keywords: European human rights law; principle of equality; public policy; fraus legis; method of recognition

Mots clés : convention européenne des droits de l'homme ; principe d'égalité ; ordre public ; fraude à la loi ; méthode de la reconnaissance

DOI: $10.14712 / 23366478.2020 .31$

\section{INTRODUCTION : DES ARRÊTS ET DÉCISIONS «AYANT TRAIT »AUX CONFLITS DE LOIS}

1. Les décisions de la Cour européenne des droits de l'homme « ayant trait » au droit international privé que l'on présentera ici ne sont pas des décisions de droit international privé. La Cour n'est (contrairement aux juridictions nationales, ou à la Cour de justice de l'Union européenne) pas une juridiction qui aurait directement compétence pour trancher des questions de droit international privé, ni d'ailleurs, plus généralement, des questions de droit allant au-delà de l'application d'un seul ensemble

\footnotetext{
* Avocat, professeur à l’Université du Luxembourg. Secrétaire général du GEDIP.
} 
normatif, la Convention européenne des droits de l'homme et ses protocoles additionnels. Mais la Cour européenne a potentiellement vocation à intervenir - en le faisant, comme le rappelle à présent le Protocole additionnel no 15 à la Convention européenne, dans le respect du principe de subsidiarité et en respectant la marge d'appréciation dont bénéficient les Etats cocontractants ${ }^{1}$ - dans le contrôle, par rapport à la Convention, du résultat de l'application des règles nationales (ou européennes au sens de : règles de l'Union européenne ${ }^{2}$ ) de droit international privé.

C'est que les décisions et arrêts de la Cour européenne des droits de l'homme peuvent intervenir à propos de n'importe quel domaine de la réalité ou presque. ${ }^{3}$ Comme la formulation et l'administration des règles du droit international privé font partie de l'activité des pouvoirs publics des Etats contractants, et comme elles sont susceptibles de porter des atteintes réelles (ou du moins alléguées par les requérants) aux droits de l'homme des personnes qui se relèvent de la juridiction des Etats contractants, elles sont concernées elles aussi par le contrôle de la Cour.

2. Le contrôle par rapport aux droits de l'homme ne peut pas réellement nuire aux méthodes générales du droit international privé. Ces méthodes survivront à leur confrontation aux droits de l'homme et au contrôle de leurs résultats par une juridiction internationale qui n'est pas spécialisée en droit international privé. ${ }^{4}$ Il peut en aller différemment de l'une ou de l'autre application de ces méthodes, et il faut s'en féliciter, car des solutions non seulement très discutables au regard des buts réels du droit international privé, mais encore attentatoires aux droits de l'homme existent parfois dans les systèmes nationaux de droit international privé.

Les décisions et arrêts issus de ce contrôle - par essence aléatoire, puisque réalisé par une juridiction qui ne peut pas s'autosaisir - sont intéressants à connaître et à systématiser, y compris dans une perspective de codification progressive du droit international privé de l’Union européenne. Il ne convient en effet évidemment pas de favoriser les

1 Le $15^{\mathrm{e}}$ Protocole a ajouté un nouveau considérant au préambule de la Convention, ainsi rédigé : «Affirmant qu'il incombe au premier chef aux Hautes Parties contractantes, conformément au principe de subsidiarité, de garantir le respect des droits et libertés définis dans la présente Convention et ses protocoles, et que, ce faisant, elles jouissent d'une marge d'appréciation, sous le contrôle de la Cour européenne des Droits de l'Homme instituée par la présente Convention ». Le Protocole n'est pas encore entré en vigueur ; sa ratification par la Bosnie-Herzégovine et l'Italie n'est pas encore intervenue. Mais de toute manière, le texte en question correspond à la jurisprudence existante de la Cour.

2 Cf. en particulier l'arrêt Avotingš c. Lettonie du 23 mai 2016 [GC], no 17502/07.

3 Dans un article publié en 2011, j'en avais proposé une preuve empirique, en proposant aux lecteurs de voir ce qui serait le résultat d'une recherche sur le système « Hudoc » mis à disposition du public sur www. echr.coe.int, qui utiliserait comme terme de recherche le mot « druide » (ou plutôt son équivalent anglais, « druid »). Le résultat en était qu'il y avait deux décisions et un arrêt faisant apparaitre le terme « druid »; les deux décisions avaient trait à des requêtes de druides dirigées contre le Royaume-Uni, à propos des règlements de police s'appliquant au site de Stonehenge qui portaient indûment atteinte, selon les requérants, au libre exercice de la religion druidique : cf. Private International Law Topics Before the European Court of Human Rights - Selected Judgments and Decisions (2010-2011). Yearbook of Private International Law, 2011, 13, pp. 37 et seq., spécialement note 2.

4 La même observation est exacte en ce qui concerne le contrôle par une juridiction constitutionnelle nationale. Cf. d'une manière générale « Droits de l'homme, droits fondamentaux et droit international privé ». Recueil des cours, 2005, vol. 318, p. 22, note 9 (où il est observé que ce contrôle a même un avantage notable : l'absence de spécialisation en droit international privé de la juridiction chargée de contrôler le respect des droits de l'homme lui permet d'avoir un regard pour ainsi dire naïf, et qui peut être plus perspicace que le regard de ceux qui sont plus routiniers de la matière). 
conflits de normes ; la codification doit s'efforcer de respecter le droit international des droits de l'homme liant l'ensemble des Etats membres, et qui inspire au demeurant la Charte des droits fondamentaux de l'Union européenne. ${ }^{5}$

3. L'activité de la Cour au cours des années activité dépend des requêtes dont la Cour est saisie. Parmi les sous-matières du droit international privé général, c'est le domaine de la coopération en matière de lutte contre l'enlèvement international d'enfants - par application de la Convention de La Haye du 25 octobre 1980 sur les aspects civils de l'enlèvement international d'enfants, et à l'intérieur de l'Union européenne par application des règlements Bruxelles-II, Bruxelles IIbis et (à l'avenir) de la refonte de ce dernier, opérée par le règlement (UE) 2019/1111 - qui est celui dans lequel l'activité jurisprudentielle est la plus intense, simplement parce que le nombre de saisines est le plus élevé ; la jurisprudence est assez casuistique mais s'oriente autour des principes arrêtés dans deux arrêts de Grande Chambre, les arrêts Neulinger et Shuruk c. Suisse ${ }^{6}$ et X. c. Lettonie. $^{7}$

S'agissant de la compétence judiciaire internationale, il n'y a en revanche pas de grands thèmes prédominants, mais plutôt des solutions individuelles qui ont été adoptées dans des arrêts de la Cour européenne. Ces arrêts se sont jusqu'à présent concentrés (du fait, à nouveau, de la nature des requêtes dont la Cour avait été saisie) sur l'aspect de garantie de l'effectivité de l'accès aux tribunaux pour les demandeurs ${ }^{8}$ plutôt que sur la protection des défendeurs contre les affirmations de compétence exorbitantes pouvant émaner des tribunaux des Etats contractants.

Dans le domaine de la reconnaissance et de l'exécution des jugements étrangers, un Etat contractant peut méconnaître la Convention en refusant d'exécuter un jugement étranger pour des motifs non reconnus comme suffisants au regard du droit à l'exécution effective d'une décision obtenue, ${ }^{9}$ ou alors en l'exécutant malgré le fait que les droits garantis par la Convention ont été méconnus lors de la procédure devant le juge d'origine. Les deux hypothèses apparaissent périodiquement dans la jurisprudence de la Cour ; la deuxième de ces hypothèses est une cause de tension entre le droit européen des droits de l'homme et le droit de l'Union européenne qui met en œuvre, au moins partiellement, une politique fondée sur la confiance mutuelle entre Etats membres et comportant l'exécution des jugements émanant des tribunaux d'autres Etats contractants, sans contrôle dans l'Etat membre de l'exécution de ces jugements. ${ }^{10}$

5 Cf. l'article 52, paragraphe 3 de la Charte : « Dans la mesure où la présente Charte contient des droits correspondant à des droits garantis par la Convention européenne de sauvegarde des droits de l'Homme et des libertés fondamentales, leur sens et leur portée sont les mêmes que ceux que leur confère ladite convention. Cette disposition ne fait pas obstacle à ce que le droit de l'Union accorde une protection plus étendue ».

6 Arrêt du 6 juillet 2010, $\mathrm{n}^{\circ} 41615 / 07$.

7 Arrêt du 26 novembre 2013, no 27853/09.

8 Arlewin c. Suède, arrêt du 1er mars 2016, no 22302/10. Par ailleurs, un arrêt de la Grande Chambre de la Cour, l'arrêt Nä̈t-Liman c. Suisse, arrêt du 15 mars 2018, no 51357/07, a considéré comme justifié le refus de la Suisse d'accepter la compétence de ses tribunaux pour connaître de faits de torture dont avait été victime un Tunisien réfugié en Suisse, et ceci malgré l'impossibilité d'obtenir la compétence des juridictions tunisiennes. Comme on le sait, l'arrêt Naït-Liman a été très critiqué.

9 Cf. « Enforcement as a Fundamental Right ». Nederlands Internationaal Privaatrecht, 2014, pp. 540 et seq.

10 Sur ce point, cf. « Le rôle du politique en droit international privé ». Recueil des cours, 2019, t. 402, pp. $295-310$. 
4. Ce que je me propose de présenter ici est la jurisprudence de la Cour européenne des droits de l'homme au cours de la dernière décennie dans un autre domaine que ceux qui viennent d'être mentionnés, celui des conflits de lois. Le conflit de lois est l'une des matières centrales du droit international privé, et reste (du moins à mon avis) la matière la plus caractéristique du mode très particulier de raisonner qui caractérise le droit international privé.

Là encore, ce n'est pas à la Cour européenne des droits de l'homme qu'il appartiendrait de définir des règles de conflits ou de se prononcer sur telle ou telle technique du droit international privé. Je montrerai au contraire, à l'aide de sa jurisprudence des dix dernières années, qu'elle est respectueuse de la compétence des Etats contractants à cet égard. Mais elle se réserve un contrôle, qu'elle a exercé jusqu'à présent dans quatre domaines : la non-discrimination par la règle de conflit (II), le jeu de l'ordre public d'éviction (III), celui de l'exception de fraude à la loi (IV) et, enfin - mais c'est un last not least - celui du choix méthodologique ou plutôt du résultat de ce choix, ${ }^{11}$ entre la méthode de la reconnaissance et la méthode de l'application de la règle de conflit du for à des situations constituées en dehors du for $(\mathrm{V})$.

\section{LE CONTRÔLE EUROPÉEN DE LA NON-DISCRIMINATION PAR LA RÈGLE DE CONFLIT}

5. La règle de conflit de lois est essentiellement un mécanisme de restriction de l'applicabilité des normes de droit matériel du for à certaines situations : celles que le facteur de rattachement de la règle de conflit localise dans l'Etat du for. Pour toutes les autres situations, non localisées dans le for, ce sera une loi étrangère, désignée conformément au système de conflit du for, qui sera appelée à les régir. Lorsque ce système de conflit est conforme à la tradition européenne dominante, la règle de conflit sera multilatérale et procédera elle-même à la désignation de la loi étrangère qui s'appliquera devant les autorités du for à ces situations, au lieu du droit matériel du for. Cette description, élémentaire, du fonctionnement des règles de conflit a parfois donné lieu à un soupçon qui a été (trop ?) rapidement écarté par les spécialistes du droit international privé : le traitement matériel différencié des deux types de situation, dès lors que la solution qui résulte de la loi du for n'est pas identique à la solution du droit étranger, alors que les deux situations sont similaires sauf par leur localisation, n'est-il pas discriminatoire ? $?^{12}$

La discrimination potentielle par la règle de conflit, à laquelle la Cour européenne a consacré la décision et l'arrêt que l'on présentera ici, relève de la violation du principe général de l'égalité devant la loi. Elle se distingue en cela de l'incidence de principes

11 Puisque, là encore, la Cour européenne des droits de l'homme s'est toujours gardée d'imposer un choix méthodologique aux Etats contractants. Il lui arrivera, en revanche, de censurer certains résultats de l'application de la méthode classique de la règle multilatérale de conflit de lois.

$12 \mathrm{Cf}$. «Sur la question de la discrimination inhérente aux règles de conflit de lois. Développements récents et interrogations permanentes ». Dans : CORTESE, B. (ed.). Studi in onore di Laura Picchio Forlati. Torino : G. Giappichelli Editore, 2014, pp. 195 et seq., dont est également reprise la description des affaires Ammdjadi c. Allemagne et Harroudj c. France ci-dessous. 
spécifiques de non-discrimination ayant une nature particulière et un poids politique particulier, spécialement l'égalité entre hommes et femmes. ${ }^{13}$

La question peut sembler appeler une réponse extrêmement simple : il n'y a pas de discrimination dans pareil cas. Du moins en va-t-il ainsi selon un argument logique, a priori convaincant, dans le sens de la non-pertinence d'une vérification des règles de conflit par rapport au principe général d'égalité devant la loi : c'est qu'il n'y a pas de traitement inégal du fait de l'application d'une règle de conflit unique à toutes les situations. Prenons l'exemple le plus important, celui du rattachement du statut personnel à la nationalité des personnes. La règle de conflit - élément de l'ordre juridique du for et par conséquent susceptible d'être confrontée au principe d'égalité qui s'impose à l'intérieur de ce même ordre juridique - a pour seul contenu d'indiquer la loi applicable au fond ; et si le droit matériel du for, qui s'applique au statut personnel des nationaux du for, prévoit à leur profit un avantage, la règle de conflit n'empêche en rien les nationaux étrangers de bénéficier du même avantage, à condition qu'il soit prévu par leur propre loi nationale. La règle de conflit, quant à elle, est la même pour tous : tous seront jugés seront leur loi nationale. Il n'y a donc pas de traitement différencié et a fortiori pas de discrimination, celle-ci étant le traitement différencié non justifié. ${ }^{14}$ Seules des règles de conflit qui différencient selon des critères non seulement irrationnels, mais absolument inacceptables peuvent, selon cette conception, être utilement confrontées au principe d'égalité. Il peut en aller autrement, bien entendu, lors de l'application des règles du droit interpersonnel, règles de conflit internes à un ordre juridique plurilégislatif - la Grèce en est un exemple avec l'application, par les juridictions grecques, de la charia aux membres de la minorité musulmane de Thrace, comme devait le montrer l'arrêt Molla Sali c. Grèce de la Cour européenne. ${ }^{15}$ L'explication de cette exception sera que tant le droit commun grec que le droit musulman font partie de l'ordre juridique de la Grèce : le critère de répartition des normes est susceptible de contrôle au regard du principe général d'égalité, contrairement aux règles du droit international privé déclarant applicables tantôt les normes issues de l'ordre juridique du for, tantôt celles issues d'un ordre juridique étranger.

13 Une affaire complexe, faisant intervenir le droit international privé suisse et la réglementation suisse de droit matériel en matière d'attribution du nom après mariage, a été tranchée par l'arrêt de la Cour du 9 novembre 2010, Losonci Rose et Rose c. Suisse, n 664/06. Cet arrêt décide que le fait de priver M. Losonci de la possibilité de choisir la loi applicable était discriminatoire en raison de son sexe (si une femme mariée avait demandé à changer de nom, le droit suisse 1'y aurait admis dans une situation symétrique), en rappelant que « seules des considérations très fortes peuvent amener à estimer incompatible avec la Convention une différence de traitement fondée exclusivement sur le sexe » (par. 41). L'affaire Losonci-Rose est trop particulière pour que l'on puisse en tirer des conclusions à portée générale.

$14 \mathrm{Cf}$. le raisonnement conduit, avec une implacable logique, par SONNENBERGER, H.-J. Münchener Kommentar zum Bürgerlichen Gesetzbuch. 5e éd. München: C. H. Beck, 2010, Einleitung IPR, no 341, note 1073 : le principe de non-discrimination ne peut viser que l'auteur de la règle de conflit; dès lors, une critique ne serait pertinente que si la règle de conflit prévoyait un rattachement différent pour des mêmes situations de fait - ce qui n'est précisément pas le cas en cas d'un rattachement uniforme, tel le rattachement à la nationalité.

15 Arrêt du 19 décembre 2018 [GC], requête no 20452/14. La Cour y juge (par. 157) que « [r]efuser aux membres d'une minorité religieuse le droit d'opter volontairement pour le droit commun et d'en jouir non seulement aboutit à un traitement discriminatoire, mais constitue également une atteinte à un droit d'importance capitale dans le domaine de la protection des minorités, à savoir le droit de libre identification. » 
6. Mais est-il vrai que, comme le soutient cette opinion doctrinale, une règle de conflit en matière de conflits de lois d'ordres juridiques différents est soustraite à tout contrôle ? Deux affaires portées devant la Cour européenne des droits de l'homme permettent d'en douter.

La première de ces affaires, l'affaire Ammdjadi c. Allemagne ${ }^{16}$ concernait les conséquences du divorce d'un couple d'Iraniens, domiciliés en Allemagne. Lorsque l'épouse demanda le Versorgungsausgleich (péréquation des droits à pension), conséquence du divorce qui existe en droit allemand, mais qui n'existe pas en droit iranien, elle se heurta à une disposition du traité d'établissement entre l'Allemagne et l'Empire de Perse du 17 février 1929, en vertu de laquelle en matière personnelle, familiale et successorale, la loi de la nationalité commune des parties est applicable. Cette solution adoptée par le traité était moins favorable à la requérante que celle du droit international privé commun allemand (art. 17, par. 3 EGBGB), lequel permet, en fonction des circonstances, de procéder à la péréquation des droits à pension entre conjoints étrangers si ces droits ont été acquis en Allemagne. Pour la requérante, l'application du droit iranien - qui lui déniait le droit à la péréquation, solution qui avait été jugée non contraire à l'ordre public allemand par les juridictions nationales - heurtait son droit à la vie familiale (art. 8 de la Convention), mais aussi son droit à ne pas être l'objet d'une discrimination en raison de sa nationalité iranienne (art. 14 en combinaison avec l'art. 8).

La Cour rejette d'abord le grief tiré de l'art. 8, estimant que la Convention n'oblige pas les tribunaux allemands à laisser sans application le traité germano-perse en qualifiant la péréquation des droits à pension comme faisant partie de l'ordre public allemand. Elle note dans ce contexte que le droit iranien prévoit lui aussi pour une « certaine protection sociale du conjoint », prenant la forme d'aliments après divorce.

Vient ensuite l'examen du grief tiré du principe d'égalité. La Cour retient que la requérante a effectivement été traitée différemment d'autres demandeurs en péréquation, auxquels s'applique le droit matériel allemand, et que cette différence de traitement est due à la nationalité iranienne de la requérante et de son conjoint. Si elle n'a pas pour autant conclu à l'existence d'une discrimination, c'est que la règle de conflit en question - tout en apparaissant quelque peu inopportune à la Cour qui lui préférerait en l'occurrence apparemment un rattachement à la résidence habituelle des parties - n'est pas pour autant sans justification « objective et raisonnable », si bien que rien ne s'oppose à son maintien. La Cour note par ailleurs que le rattachement à la nationalité a, en général, pour utilité de « protéger les liens étroits d'une personne avec son Etat national» :

« The Court finds that, especially in conflict of laws cases, the differentiation for all family issues according to nationality and not to habitual residence is a well-known principle which aims at protecting a person's close connection with his or her home country. Therefore, even though the decisiveness of the habitual residence might arguably be considered preferable with regard to pension rights, the decisiveness of a person's nationality cannot be considered to be without "objective and reasonable justification" ». ${ }^{17}$

16 Décision du 9 mars 2010, no 51625/08.

17 La décision poursuit comme suit : «In this respect, it must also be noted that the applicant had been free to choose the application of German law, together with her husband, by notarial certification ». Il semblerait que cette réflexion confonde les règles ordinaires du droit international privé allemand (article 14, para- 
Ammdjadi c. Allemagne est une décision d'irrecevabilité par laquelle la Cour motive brièvement la conclusion selon laquelle les griefs de la requérante étaient « manifestement malfondés ». Ces motifs n'en sont pas moins remarquables. La raison pour laquelle il a été conclu à l'absence de discrimination ne consistait pas dans le fait que le traité germano-perse formulait une règle de conflit uniformément applicable à toutes les personnes qui entraient dans son champ d'application (à savoir la règle de l'applicabilité de leur loi nationale, qu'elle soit allemande ou iranienne). Au contraire, la décision s'intéresse au facteur de rattachement choisi par le traité germano-perse et estime que ce facteur de rattachement est objectivement et raisonnablement justifié - ce qui, compte tenu de la marge d'appréciation que la Cour concède au législateur national, revient à le valider pour les besoins du principe d'égalité au sens de la Convention européenne des droits de l'homme.

C'est uniquement parce que la nationalité apparaît comme un facteur de distinction pertinent et raisonnable dans l'octroi entre époux allemands d'un droit à péréquation, et dans son refus entre époux iraniens, que la requête a été rejetée. Cette approche diffère de celle selon laquelle une règle de conflit unique traite par nature, quel que soit le facteur de rattachement choisi, toutes les personnes de manière identique. L'approche ainsi adoptée est une approche moins formelle et plus substantielle de la question de la discrimination par la règle de conflit de lois.

7. L'affaire Harroudj c. France a donné lieu à une motivation plus ample de la solution adoptée. ${ }^{18}$ La requérante, une ressortissante française, s'était vu confier en Algérie, par un acte de recueil légal (kafala), un enfant algérien abandonné, Hind. L'acte de recueil légal autorisait l'enfant à sortir du territoire algérien et à s'établir en France avec la requérante. Deux ans après, $\mathrm{M}^{\mathrm{me}}$ Harroudj demanda en France l'adoption plénière de l'enfant, au motif que ceci était la solution la plus conforme à " l'intérêt supérieur de l'enfant ». Mais le droit algérien, d'inspiration islamique, prohibe l'adoption, ${ }^{19}$ et une règle de conflit du droit français, l'article 370-3 du code civil, dispose que « l'adoption d'un mineur étranger ne peut être prononcée si sa loi personnelle prohibe cette institution, sauf si ce mineur est né et réside habituellement en France $» .{ }^{20}$ La requérante échoua par conséquent à obtenir en France l'adoption de l'enfant. Elle introduisit un recours devant la Cour européenne des droits de l'homme, fondé d'une part sur l'atteinte à sa vie familiale avec l'enfant qu'aurait constitué le refus de prononcer l'adoption et, d'autre part, sur la discrimination dont elle serait victime, par comparaison à une mère adoptant un enfant de statut personnel non prohibitif de l'adoption.

La Cour répond au grief tiré de la discrimination, ${ }^{21}$ qu'elle examine après le grief tiré de l'atteinte à la vie familiale (garantie par l'article 8 de la Convention), « qu'au

graphe 4, EGBGB, qui permet aux époux de choisir la loi applicable aux effets généraux de leur mariage) et les règles du traité germano-perse de 1929 qui s'appliquait en l'occurrence comme lex specialis. Mais ceci est sans importance réelle.

18 Arrêt du 4 octobre 2012, no 43631/09.

19 Aux termes de l'article 46 du Code de la famille algérien, « l'adoption est interdite par la chari'a et la loi ».

20 L'article 370-3 du Code civil est issu de la loi no 2001-111 du 6 février 2001 et est inséré au chapitre III (Du conflit des lois relatives à la filiation adoptive et de l'effet en France des adoptions prononcées à l'étranger) du titre VIII relatif à la filiation adoptive.

21 Article 14 de la Convention, en combinaison avec l'article 8. 
cœur du grief énoncé par la requérante sur le terrain de l'article 14 de la Convention se trouve l'impossibilité d'adopter l'enfant Hind en raison de sa loi personnelle. Cette question a été examinée sous l'angle de l'article 8 [...]. Dans ces conditions, la Cour estime qu'aucune question distincte ne se pose au regard de l'article 14 de la Convention et ne formule aucune conclusion séparée sur ce grief $\gg .^{22} \mathrm{Il}$ faut donc se rapporter à la réponse de la Cour au grief tiré de l'atteinte au droit à la vie familiale pour connaître la motivation jugée également applicable à la discrimination alléguée. La Cour y retient que « le refus opposé à la requérante tient en grande partie au souci du respect de l'esprit et de l'objectif des conventions internationales »-ce qui est visé étant d'une part la Convention des Nations Unies relative aux droits de l'enfant du 20 novembre 1989, dont l'article 20 met sur un même plan (et considère comme également conformes à l'intérêt supérieur de l'enfant) la kafala de droit islamique et l'adoption, ainsi que les Conventions de La Haye du 29 mai 1993 sur la protection des enfants et la coopération en matière d'adoption internationale et du 19 octobre 1996 concernant la compétence, la loi applicable, la reconnaissance, l'exécution et la coopération en matière de responsabilité parentale et de mesures de protection des enfants, qui reposent sur la même logique. Selon la Cour, « la reconnaissance de la kafala par le droit international est un élément déterminant pour apprécier la manière dont les Etats la réceptionnent dans leurs droits nationaux et envisagent les conflits de loi qui se présentent ».23

Ensuite, la Cour relève que si certaines différences entre la kafala et l'adoption sont insurmontables (en particulier absence d'effets successoraux de la kafala), il peut être remédié dans une certaine mesure aux restrictions qu'engendre l'impossibilité d'adopter l'enfant. ${ }^{24}$ Ces préalables étant exposés, la conclusion est la suivante :

«L'ensemble des éléments examinés ci-dessus fait apparaître que l'Etat défendeur, appliquant les conventions internationales régissant la matière, a institué une articulation flexible entre le droit de l'Etat d'origine de l'enfant et le droit national. La Cour relève à ce titre que le statut prohibitif de l'adoption résulte de la règle de conflit de lois de l'article 370-3 du code civil mais que le droit français ouvre des voies d'assouplissement de cette interdiction à la mesure des signes objectifs d'intégration de l'enfant dans la société française. C'est ainsi, d'une part, que la règle de conflit est écartée explicitement par ce même article 370-3 lorsque « le mineur est né et réside habituellement en France ». D'autre part, cette règle de conflit est volontairement contournée par la possibilité ouverte à l'enfant d'obtenir, dans un délai réduit, la nationalité française, et ainsi la faculté d'être adopté, lorsqu'il a été recueilli en France par une personne de nationalité française. La Cour observe à ce titre que l'Etat défendeur soutient sans être démenti que la jeune Hind pourrait déjà bénéficier de cette possibilité.

La Cour estime qu'en effaçant ainsi progressivement la prohibition de l'adoption, l'Etat défendeur, qui entend favoriser l'intégration d'enfants d'origine étrangère sans les couper immédiatement des règles de leur pays d'origine, respecte le pluralisme culturel et ménage un juste équilibre entre l'intérêt public et celui de la requérante $»{ }^{25}$

22 Par. 55.

23 Par. 50.

24 Par. 51 : « Outre la requête en concordance de nom ici acquise du fait de la filiation inconnue de l'enfant en Algérie, il est possible d'établir un testament, qui a pour effet de faire entrer l'enfant dans la succession de la requérante et de nommer un tuteur légal en cas de décès du recueillant ».

25 Par. 51. 
L'arrêt Harroudj est remarquable par l'attention qu'accorde la Cour européenne des droits de l'homme aux buts poursuivis par le droit international privé en général et au rattachement du statut personnel à la nationalité en particulier. Mais il est également évident que la Cour n'entend pas accepter telles quelles, sans examen, les solutions du droit international privé national qui soumettent la question de l'adoptabilité de l'enfant au droit algérien. Elle estime que les solutions du droit international privé français sont conciliables avec le droit à la vie familiale et donc également avec le droit à la non-discrimination, mais uniquement parce qu'elles admettent qu'à terme, «à la mesure des signes objectifs d'intégration de l'enfant dans la société française », la solution substantielle de la loi française (l'adoptabilité) remplace la solution substantielle restrictive du droit algérien, et que le rattachement à la nationalité algérienne n'est pas immuable en cas d'acquisition ultérieure de la nationalité française.

Le grief de discrimination n'est en conséquence pas rejeté au motif que l'inadoptabilité de l'enfant n'est pas une solution du droit matériel français et qu'il est impossible de la comparer, aux fins de vérification du respect de l'égalité des personnes devant la loi, à la solution favorable à l'adoptabilité qui est celle du droit civil français. Il n'est pas non plus écarté parce que la règle de conflit, qui désignait dans le cas de Mme Harroudj le droit algérien, opère sans discrimination puisqu'elle se borne à renvoyer, sans distinction, au statut personnel de l'enfant. Ceci n'est pas le raisonnement de la Cour, qui accepte au contraire expressément de comparer la solution restrictive du droit algérien avec la solution du droit français. Ce faisant, elle semble montrer qu'elle accepte de prendre en considération, aux fins de confrontation au principe de non-discrimination, les deux solutions matérielles que les autorités françaises peuvent avoir à appliquer, selon la nationalité de l'enfant : adoptabilité ou inadoptabilité.

C'est dire aussi que la Convention aurait pu être méconnue si le système français de conflit de lois s'était montré excessivement, déraisonnablement, rigide et n'avait admis aucun assouplissement au rattachement de la question de l'adoptabilité au droit de l'Etat d'origine de l'enfant. En somme, derrière la solution adoptée par la Cour il y a l'idée qu'il existe, non pas en droit civil français mais en France quand même, deux solutions de droit matériel : la solution de l'adoptabilité, pour les enfants de statut personnel non prohibitif, et la solution de l'inadoptabilité pour les enfants de statut personnel prohibitif. Le champ d'application de ces deux solutions est déterminé par la règle de conflit française. Et donc la règle de conflit est susceptible d'un contrôle au regard de la règle de non-discrimination, de même que l'application du droit algérien désigné par la règle de conflit française est susceptible d'un contrôle au regard du droit à la vie familiale.

8. La décision Ammdjadi c. Allemagne et l'arrêt Harroudj c. France reposent ainsi sur une analyse fondamentalement différente de celle qui écarte, comme logiquement inconcevable, toute violation du principe général d'égalité par une règle de conflit multilatérale. Certes, ils ne concluent pas à la discrimination et montrent au contraire une grande tolérance à l'égard du contenu des règles de conflit, et notamment à l'égard de la question du rattachement du statut personnel à la nationalité ou à la résidence. ${ }^{26}$ Mais ils reposent implicitement sur une approche théorique particulière. Pour l'expliciter,

26 Cette tolérance apparaît tout particulièrement dans la décision Ammdjadi c. Allemagne, dans laquelle la Cour semble estimer qu'un rattachement à la résidence habituelle aurait été préférable, mais dans laquelle 
on dira que c'est la coexistence de deux solutions matérielles différentes - présence ou absence d'un droit à la péréquation des droits à pension, adoptabilité ou inadoptabilité des enfants - qui justifie que la question de l'éventuelle discrimination soit posée en principe : coexistence à l'intérieur de l'ordre juridique des Etats contractants concernés et avec détermination du champ d'application des deux solutions matérielles par les règles de conflit de ces Etats contractants. C'est parce qu'elles apparaissent, du fait de leur désignation par les règles de conflit allemande ou française, comme faisant partie des solutions qui sont acceptées dans les ordres juridiques allemand et français, que les solutions iraniennes ou algériennes deviennent susceptibles de contrôle au regard de la Convention européenne des droits de l'homme, et que la répartition des solutions de droit matériel entre leurs destinataires, opérée par la règle de conflit, peut être confrontée au principe d'égalité devant la loi. ${ }^{27}$

\section{LE CONTRÔLE EUROPÉEN DU JEU DE L'ORDRE PUBLIC D'ÉVICTION}

9. Certes censé être un mécanisme d'exception, d'emploi rare, dans la méthodologie classique du droit des conflits de lois, le recours à l'ordre public d'éviction n'en est pas moins un mécanisme fondamental auquel aucun Etat ne peut renoncer complètement. La Cour européenne l'a expressément reconnu, spécialement dans les arrêts Labassee c. France et Mennesson c. France, à propos de la non-reconnaissance en France de gestations pour autrui effectuées aux Etats-Unis d'Amérique. Dans ces arrêts, elle fait le lien entre le contenu de l'ordre public et les choix démocratiques des différents législateurs nationaux ; ce faisant, elle reconnaît le principe de la possibilité pour la jurisprudence des tribunaux des Etats contractants de voir dans certains des principes du droit national des principes par lesquels s'exprime l'ordre public international au sens du droit international privé dans les Etats en question - tout en retenant que la Convention européenne ne donne pas carte blanche au législateur national :

« La Cour constate que [l'approche restrictive de la jurisprudence française] se traduit par le recours à l'exception d'ordre public international, propre au droit international privé. Elle n'entend pas la mettre en cause en tant que telle. Il lui faut néanmoins vérifier si en appliquant ce mécanisme en l'espèce, le juge interne a dûment pris en compte la nécessité de ménager un juste équilibre entre l'intérêt de la collectivité à faire en sorte que ses membres se plient au choix effectué démocratiquement en son sein et l'intérêt des requérants - dont l'intérêt supérieur des enfants - à jouir pleinement de leurs droits au respect de leur vie privée et familiale $\gg .^{28}$

elle n'en juge pas moins que le rattachement à la nationalité n'est pas sans justification objective et raisonnable.

27 Voir la partie III de l'article précité, aux Studi in onore di Laura Picchio Forlati, pour une discussion des raisons de droit public de cette possibilité de confronter des règles de conflit ordinaires de droit international privé à ce principe structurant de l'ordre juridique qu'est le principe d'égalité.

28 Arrêts du 26 avril 2014, nos 65041/11 et 65192/11, par. 63 du premier arrêt et par. 84 du second. 
10. Dans certains cas, le résultat de ce contrôle européen sera un satisfecit délivré aux Etats contractants :

L'arrêt Z.H. et R.H. c. Suisse ${ }^{29}$ rejette ainsi une requête introduite par des ressortissants afghans qui ont été mariés religieusement en Iran à un moment où l'homme était âgé de dix-huit ans et son épouse (qui est aussi sa cousine) était âgée de quatorze ans. Les deux ont demandé l'asile en Suisse. La décision des autorités compétentes était de permettre à l'épouse de rester en Suisse, mais d'expulser le mari vers l'Italie, l'Italie étant l'Etat compétent par application du règlement de Dublin qui s'applique à la Suisse en vertu de l'accord d'association helvético-européen de 2004. ${ }^{30}$ Pour refuser de reconnaître le droit du mari à la vie familiale, le tribunal administratif fédéral décida que le prétendu mariage religieux des deux requérants ne pouvait pas être reconnu en Suisse, étant donné $1^{\circ}$ son illégalité au regard du code civil afghan (âge minimum du mariage : quinze ans en ce qui concerne les femmes) et $2^{\circ}$ qu'en tout état de cause, indépendamment même du droit afghan applicable, le mariage des requérants était manifestement incompatible avec l'ordre public suisse : les relations sexuelles avec un enfant en dessous de l'âge de seize ans constituent une infraction au regard du code pénal suisse. Comme le mariage n'est pas reconnu en Suisse, le requérant masculin ne peut pas être reconnu comme membre de la famille de son « épouse » et ne bénéficie dès lors pas des droits reconnus par le règlement de Dublin au profit des membres de la famille.

La requête, fondée sur la violation de l'article 8 de la Convention (droit à la vie familiale), est rejetée par la Cour, qui confirme la conformité avec la Convention des motifs de l'arrêt du tribunal administratif fédéral :

«The Court does not see any reason to depart from the findings of the [Federal Administrative Court] in this respect. Article 8 of the Convention cannot be interpreted as imposing on any State party to the Convention an obligation to recognise a marriage, religious or otherwise, contracted by a 14 year old child $» .{ }^{31}$

L'intervention de l'ordre public suisse contre le mariage d'une jeune fille de quatorze ans est par conséquent conforme aux valeurs véhiculées par la Convention européenne des droits de l'homme, ce qui justifie cette exception à l'obligation de reconnaitre le mariage célébré en Iran.

11. Dans d'autres cas, la Cour juge que l'invocation de l'ordre public national ne peut justifier l'ingérence dans un droit garanti par la Convention. La raison peut être de deux ordres. Soit c'est le type d'ordre public qui est considéré en lui-même comme insuffisamment fort pour légitimer réellement l'ingérence ; l'exemple-type en est un ordre public excentrique comme celui qui était en cause dans l'affaire Negrepontis-Giannisis c. Grèce que nous examinerons en premier lieu. Soit le recours à l'ordre public n'apparaît comme illégitime au regard des garanties de la Convention que parce que le fait

29 Arrêt du 8 décembre 2015, no 60119/12.

30 Ultérieurement, la requête d'asile des deux requérants a été acceptée, mais les requérants ont maintenu leur requête dans l'espoir de voir sanctionner la violation passée de leurs droits par la Suisse.

31 Par. 44. 
d'avoir recours à lui est disproportionné ; ceci est illustré par la saga de la non-reconnaissance des gestations pour autrui à l'étranger, deuxième cas que nous examinerons.

12. L'affaire Negrepontis-Giannisis c. Grèce $e^{32}$ met en scène un moine grec, évêque de l'Eglise orthodoxe à Detroit aux Etats-Unis d'Amérique, qui y adopte son neveu majeur, lui-même résident aux Etats-Unis depuis trois ans pour ses études universitaires. Le fils adoptif ajoute le nom de son père adoptif à son propre nom patronymique ; il rentre, au terme de ses études, en Grèce et entretient, pendant 14 ans, des liens qui correspondent à une filiation adoptive avec son oncle, lequel revient lui-même en Grèce deux ans avant son décès. Le moine - autre aspect par lequel il se singularise - laisse une fortune personnelle suffisamment considérable pour que le fils adoptif et les frères et sœurs du défunt se lancent dans une bataille judiciaire sur son partage : le fils adoptif se prétend seul héritier, mais l'une de ses tantes saisit le tribunal d'Athènes pour qu'il soit déclaré que le défunt, en tant que moine, n'avait pas la capacité pour adopter un enfant et qu'en conséquence, le jugement d'adoption américain ne peut être reconnu en Grèce.

La Cour de cassation grecque, formation plénière, est saisie du cas. Elle consulte les décisions des conciles du premier millénaire et les traditions sacrées, auxquelles se réfère l'article 3 de la Constitution grecque ${ }^{33}$ et auxquelles la Cour de cassation reconnaît des effets civils dans l'ordre juridique grec. Elle en déduit qu'en droit civil, un moine ne peut pas adopter et juge que la reconnaissance d'un jugement étranger prononçant une adoption par un moine orthodoxe « se heurte à l'ordre public international de l'article 33 du code civil et n'est pas autorisée ». Les textes ainsi appliqués sont très anciens, ils remontent aux VII et IX ${ }^{\mathrm{es}}$ siècles. Dans une opinion dissidente, des membres de la Cour de cassation font valoir que les règles canoniques sur les effets civils de l'incapacité des moines à adopter ne pourraient plus être reconnus en droit grec, la preuve en étant que lors de l'institution du mariage civil (en 1982), l'article 1364 du Code civil (lequel interdisait le mariage des moines) a été abrogé, ce qui montrerait que le législateur estime ne plus devoir tenir compte des incapacités résultant des règles canoniques. Mais l'opinion de la majorité des juges est différente, et la reconnaissance de l'adoption du requérant a été refusée comme étant contraire à l'ordre public.

Cette invocation d'un ordre public «d'après les conceptions religieuses et morales de l'Eglise Orthodoxe Orientale du Christ » ne résiste pas au contrôle opéré par la Cour européenne des droits de l'homme, qui décide que du fait du refus de reconnaître les effets en Grèce du jugement d'adoption américain, la Grèce a méconnu les droits du requérant - droits substantiels (droit au respect de la vie familiale, droit au respect des biens, droit à la non-discrimination) et droits procéduraux (droit au procès équitable, dont découle un droit à l'exécution internationale des jugements, même obtenus dans un Etat non contractant). Les textes dont s'inspire, en l'espèce, l'ordre public grec sont

32 Arrêt du 3 mai 2011, no 56759/08.

33 «La religion dominante en Grèce est celle de l'Eglise Orthodoxe Orientale du Christ. L'Eglise Orthodoxe de Grèce, reconnaissant pour Chef Notre Seigneur Jésus-Christ, est indissolublement unie, quant au dogme, à la Grande Eglise de Constantinople et à toute Eglise chrétienne du même dogme, observant immuablement, comme celles-ci, les saints canons apostoliques et synodiques ainsi que les saintes traditions. [...]» (trad. fr. dans : DELPÉRÉE, F. - VERDUSSEN, M. - BIVER, K. (dir.). Recueil des constitutions européennes. Bruxelles: Bruylant, 1994, p. 344). 
en effet trop anciens et trop peu en phase avec l'évolution de la société pour pouvoir sérieusement être opposés à la reconnaissance d'une adoption qui a produit ses effets pendant plus de dix ans. L'abrogation de l'article 1364 du Code civil en est la preuve. Par conséquent, « la Cour considère que les motifs avancés par la Cour de cassation pour refuser de reconnaître l'adoption du requérant ne répondent pas à un besoin social impérieux. Ils ne sont donc pas proportionnés au but légitime poursuivi en ce qu'ils ont eu pour effet la négation du statut de fils adoptif du requérant ». ${ }^{34}$ Bien que la Cour fasse référence au caractère disproportionné de l'invocation de l'ordre public dans l'arrêt de la Cour de cassation, elle ne prend visiblement pas au sérieux les motifs mêmes d'ordre public de l'arrêt de la Cour de cassation grecque : elle va jusqu'à affirmer « que les textes sur lesquels la Cour de cassation s'était fondée étaient de nature ecclésiastique, dataient du 7ème et 9ème siècle et étaient interprétés par la Cour de cassation d'une manière qui ne correspondait pas au droit positif existant au moment des faits et reflété par l'article 3 de la loi 1250/1982. Cette disposition abrogeait un article du code civil qui interdisait aux moines de se marier $\gg .{ }^{35}$

13. Le cas des gestations pour autrui est différent. Les principes d'ordre public international qui peuvent s'opposer dans certains Etats contractants à la reconnaissance du lien de filiation des parents d'intention à l'égard des enfants ${ }^{36}$ ne sont pas, comme les principes qui intervenaient dans l'affaire Negrepontis-Giannisis, des principes religieux trop particularistes et trop anciens pour pouvoir être pris au sérieux dans une société pluraliste moderne. Ce sont, au contraire, des principes démocratiquement adoptés par les législateurs au vu du nouveau mode d'établir une parentalité qui résute du développement des formes de gestation pour autrui. C'est ce qui explique que la condamnation de la non-reconnaissance de la gestation pour autrui, là où cette condamnation est intervenue, ${ }^{37}$ a été bien plus mesurée et plus respectueuse de la marge d'appréciation nationale.

Dans la double affaire Mennesson c. France ${ }^{38}$ et Labassee c. France, ${ }^{39}$ il s'agissait du refus de la transcription des actes de naissance établis au moyen de jugements cali-

34 Par. 76.

35 Par. 102. Cette appréciation est en réalité étonnante et, pour le dire clairement, excessive, puisqu'on peut raisonnablement estimer que la formation plénière de la Cour de cassation grecque sait, (encore) mieux que la Cour européenne des droits de l'homme, quel est le contenu du droit civil grec. Ce type de motif ne devrait être utilisé qu'en cas de motivation manifestement arbitraire d'une décision judiciaire.

36 Y compris, comme dans la solution originairement adoptée par la Cour de cassation française dans un but de " prévention générale », par la non-reconnaissance du lien de filiation entre les enfants et leur père d'intention qui était également leur père biologique, et dont les gamètes avaient été utilisés lors de la conception des enfants.

37 Il y a des exceptions : voir infra, $\mathrm{n}^{\circ} 15$, l'arrêt de la Grande Chambre de la Cour dans l'affaire Paradiso et Campanelli c. Italie; voir aussi la décision D. et autres c. Belgique (no 29176/13, du 8 juillet 2014) qui a validé le système belge de délivrance de documents de voyage aux enfants nés d'une GPA en Ukraine : étant donné qu'en définitive, après l'intervention de la justice belge, le document de voyage avait été délivré aux enfants, la Cour a estimé que les difficultés administratives antérieures ne valaient pas violation de l'article 8 de la Convention. L'ingérence initiale des autorités belges était justifiée (par. 52) : « La Cour constate que le refus initial des autorités belges d'autoriser la venue de A. sur le territoire national était motivé par la nécessité de vérifier si les législations belge et ukrainienne étaient respectées. L'ingérence était donc justifiée par des objectifs de prévention des infractions pénales, en particulier de lutte contre la traite des êtres humains. Or la Cour a déjà reconnu l'importance et la nécessité de lutter contre ce phénomène (Rantsev c. Chypre et Russie, no 25965/04, § 278, CEDH 2010 (extraits)) ».

38 Arrêt du 26 avril 2014, nº 65192/11.

39 Arrêt du 26 avril 2014, no 65041/11. 
forniens (affaire Mennesson) ou du Minnesota (affaire Labassee) pour des enfants nés par gestation pour autrui aux Etats-Unis, les « parents d'intention » étant français et résidant en France. Dans les deux cas, les enfants étaient les enfants biologiques de leur père français, mais n'avaient aucun lieu de filiation biologique avec leur mère d'intention française (GPA avec don d'ovocyte).

La Cour de cassation française, appliquant avec rigueur l'exception d'ordre public, avait jugé qu'étant donné que le droit français prohibe le recours à la gestation pour autrui, la reconnaissance des jugements américains, y compris en tant qu'ils ne faisaient que refléter la réalité biologique de la filiation paternelle, ne pouvait être obtenue en France. Sur recours des couples concernés et de leurs enfants, la Cour européenne adopte une solution nuancée. Celle-ci revient à affirmer, au nom de la marge nationale d'appréciation, le droit de la France de ne pas reconnaître le lien de filiation entre les enfants et leurs mères d'intention, qui ne correspond pas à la réalité biologique ; en revanche, la non-reconnaissance de la filiation paternelle constitue une violation non pas du droit des parents et des enfants à la vie familiale, mais du droit des enfants à leur identité, dont fait partie leur filiation paternelle. Les motifs les plus significatifs sont les suivants. D'abord l'affirmation de l'existence d'une marge d'appréciation ample :

«Cette absence de consensus [entre les différents Etats membres du Conseil de l'Europe sur le traitement juridique de la GPA] reflète le fait que le recours à la gestation pour autrui suscite de délicates interrogations d'ordre éthique. Elle confirme en outre que les États doivent en principe se voir accorder une ample marge d'appréciation, s'agissant de la décision non seulement d'autoriser ou non ce mode de procréation mais également de reconnaître ou non un lien de filiation entre les enfants légalement conçus par gestation pour autrui à l'étranger et les parents d'intention ». ${ }^{40}$

Le juste équilibre est respecté, selon la Cour, en ce qui concerne l'ingérence dans le droit au respect de la vie familiale des parents et des enfants. ${ }^{41}$ En revanche, le droit des enfants au respect de leur identité est méconnu (droit à la constatation de leur filiation ; droit à la nationalité ; droit à la succession ${ }^{42}$ ), du moins à l'égard du père qui est leur père biologique :

«99. [...] les effets de la non reconnaissance en droit français du lien de filiation entre les enfants ainsi conçus et les parents d'intention ne se limitent pas à la situation de ces derniers, qui seuls ont fait le choix des modalités de procréation que leur reprochent les autorités françaises : ils portent aussi sur celle des enfants eux-mêmes, dont le droit au respect de la vie privée, qui implique que chacun puisse établir la substance de son identité, y compris sa filiation, se trouve significativement affecté. [...]

100. Cette analyse prend un relief particulier lorsque, comme en l'espèce, l'un des parents d'intention est également géniteur de l'enfant. Au regard de l'importance de la filiation biologique en tant qu'élément de l'identité de chacun [...], on ne saurait prétendre qu'il est conforme à l'intérêt d'un enfant de le priver d'un lien juridique de cette nature alors que la

40 Par. 79 de l'arrêt Mennesson.

41 Par. 93-94.

42 Par. 96-98. 
réalité biologique de ce lien est établie et que l'enfant et le parent concerné revendiquent sa pleine reconnaissance. [...]

101. Étant donné aussi le poids qu'il y a lieu d'accorder à l'intérêt de l'enfant lorsqu'on procède à la balance des intérêts en présence, la Cour conclut que le droit des troisième et quatrième requérantes au respect de leur vie privée a été méconnu ».

14. Comme la formule « cette analyse prend un relief particulier $»^{43}$ ne brille pas par sa précision juridique, restait posée la question de l'éventuelle obligation de la France de reconnaître également le lien de filiation, établi aux Etats-Unis, entre les enfants et leur mère d'intention, avec laquelle le lien biologique de filiation faisait défaut. La Cour de cassation française saisit (dans le cadre du réexamen de l'affaire Mennesson) la Cour européenne des droits de l'homme d'une demande d'avis sur la question de savoir si la France « excède la marge d'appréciation dont [elle] dispose au regard de l'article 8 de la Convention » en refusant de transcrire, dans ces circonstances, une filiation maternelle à l'égard de la mère d'intention sur ses registres de l'état civil, et, dans l'affirmative, si « la possibilité pour la mère d'intention d'adopter l'enfant n'est pas suffisante au regard des exigences de la Convention $» .{ }^{44}$ En réponse, la Grande Chambre de la Cour européenne des droits de l'homme a confirmé, dans son avis consultatif du 10 avril 2019, « relatif à la reconnaissance en droit interne d'un lien de filiation entre un enfant né d'une gestation pour autrui pratiquée à l'étranger et la mère d'intention, demandé par la Cour de cassation française $»,{ }^{45}$ qu'eu égard à la marge d'appréciation dont disposait la France au regard de la Convention, il était effectivement suffisant d'admettre l'adoption de l'enfant par sa mère d'intention, « dès lors que ses conditions sont adaptées et que ses modalités permettent une décision rapide, de manière à éviter que l'enfant soit maintenu longtemps dans l'incertitude juridique quant à ce lien. Il va de soi que ces conditions doivent inclure une appréciation par le juge de l'intérêt supérieur de l'enfant à la lumière des circonstances de la cause $» .46$

La solution adoptée par l'avis de la Grande Chambre dans l'affaire Mennesson a par la suite été confirmée par la décision de la Cour dans l'affaire C. et E. c. France ${ }^{47}$ qui a rejeté, comme irrecevable pour défaut manifeste de fondement, une requête contre la France d'un couple de parents d'intention qui faisait grief à la France d'avoir accepté de transcrire la filiation paternelle des enfants (conforme à la réalité biologique), mais non leur filiation à l'égard de leur mère d'intention; il a été jugé dans ce contexte que

« ce n'est pas imposer aux enfants concernés un fardeau excessif que d'attendre des requérants qu'ils engagent maintenant une procédure d'adoption à cette fin. [...] il résulte des éléments produits par le Gouvernement que la durée moyenne d'obtention d'une décision n'est que de 4,1 mois en cas d'adoption plénière et de 4,7 mois en cas d'adoption simple $»,{ }^{48}$

43 Cf. le paragraphe 100 de l'arrêt.

44 Ass. plén., 5 octobre 2018, no 10-19053.

45 Demande $n^{\circ}$ P16-2018-001.

46 Par. 54.

47 Décision du 19 novembre 2019 , n ${ }^{\text {os }} 1462 / 18$ et $17348 / 18$.

48 Par. 43. 
si bien que le refus des autorités françaises de transcrire les actes de naissance étrangers des enfants requérants sur les registres de l'état civil français pour autant qu'ils désignent la mère d'intention comme étant leur mère n'était pas disproportionné. La même solution a été adoptée par l'arrêt $D$. $c$. France 49 à propos d'une situation où la mère d'intention était également la mère génétique (mais avait eu recours à une père porteuse pour donner naissance à l'enfant) ; il a été jugé par la Cour qu'elle pouvait également être renvoyée à une procédure d'adoption.

La Cour de cassation française, qui avait pendant très longtemps adopté une position « bioconservatrice » en matière de gestation pour autrui, semble avoir en définitive abandonné celle-ci (ou ce qui en restait) au moment même où la Cour européenne des droits de l'homme a paru reconnaître qu'elle ne violait (plus) la Convention européenne. Au vu de l'avis consultatif de la Cour européenne des droits de l'homme, l'Assemblée plénière de la Cour de cassation a fini par juger, dans l'affaire Mennesson, qu' « en l'espèce, s'agissant d'un contentieux qui perdure depuis plus de quinze ans, en l'absence d'autre voie permettant de reconnaitre la filiation dans des conditions qui ne porteraient pas une atteinte disproportionnée au droit au respect de la vie privée [des enfants] consacré par l'article 8 de la Convention de sauvegarde des droits de l'homme et des libertés fondamentales, et alors qu'il y a lieu de mettre fin à cette atteinte, la transcription sur les registres de l'état civil de Nantes des actes de naissance établis à l'étranger [...] ne saurait être annulée $»{ }^{50}$ Des arrêts ultérieurs ont laissé entendre que la nouvelle solution, de transcription d'une filiation établie par GPA et sans lien biologique, pouvaient être préférée à la voie de l'adoption dans d'autres cas que l'affaire Menesson. ${ }^{51}$ Lassitude de l'ordre public international?

15. L'arrêt de la Grande Chambre de la Cour européenne dans l'affaire Paradiso et Campanelli c. Italie, intervenu dans une situation où (contrairement aux affaires françaises) un lien de filiation génétique n'existait avec aucun des parents d'intention ceux-ci se disaient victimes d'une erreur de la clinique russe où la conception de l'enfant avait eu lieu -, a estimé justifiée la séparation de l'enfant de ses parents d'intention sur ordre d'un tribunal italien, en jugeant notamment qu' « accepter de laisser l'enfant avec les requérants, peut-être dans l'optique que ceux-ci deviennent ses parents adoptifs, serait revenu à légaliser la situation créée par eux en violation de règles importantes du droit italien $» .52$

49 Arrêt du 16 juillet 2020, $\mathrm{n}^{\circ} 11288 / 18$.

50 Ass. plén. 4 octobre 2019, no 10-19.053.

51 Civ. $1^{\text {re }}, 18$ décembre $2019, \mathrm{n}^{\circ} 18-11.815$ et $\mathrm{n}^{\circ}$ 18-12.327, deux arrêts qui admettent la transcription de la filiation à l'égard de couples masculins résultant d'un acte de l'état civil de la Californie dans le premier cas et du Nevada dans le second, et écartent l'obligation de passer par une adoption : «Au regard des mêmes impératifs [que ceux retenus dans l'affaire Menesson dans l'arrêt de l'assemblée plénière du 4 octobre 2019] et afin d'unifier le traitement des situations, il convient de faire évoluer la jurisprudence en retenant qu'en présence d'une action aux fins de transcription de l'acte de naissance étranger de l'enfant, qui n'est pas une action en reconnaissance ou en établissement de la filiation, ni la circonstance que l'enfant soit né à l'issue d'une convention de gestation pour autrui ni celle que cet acte désigne le père biologique de l'enfant et un deuxième homme comme père ne constituent des obstacles à la transcription de l'acte sur les registres de l'état civil, lorsque celui-ci est probant au sens de l'article 47 du code civil ».

52 Arrêt du 24 janvier 2017, no 25358/12, § 215. 
16. La logique adoptée par la Cour dans les affaires Mennesson et Labassee se retrouve, mutatis mutandis, dans les affaires Orlandi et autres c. Italie, ${ }^{53}$ dans lesquelles était en cause la non-reconnaissance par les tribunaux italiens de mariages homosexuels célébrés à l'étranger (Pays-Bas, Canada) par des citoyens italiens, qui parfois étaient résidents permanents de l'Etat étranger où le mariage avait été célébré et parfois étaient des résidents italiens qui avaient profité d'un voyage à l'étranger pour y faire célébrer un mariage dont ils ne pouvaient vraisemblablement pas ignorer le caractère fragile au regard de l'ordre juridique italien. La Cour a condamné l'Italie, mais pour la même raison qu'elle avait retenue dans une autre affaire de mariage entre personnes du même sexe, purement interne à l'ordre juridique italien ${ }^{54}: c^{\prime}$ est en refusant toute forme de reconnaissance juridique aux couples du même sexe que l'Italie avait violé le droit au respect de la vie privée et familiale au sens de la Convention. L'arrêt dans les affaires Orlandi et autres n'oblige pas l'Italie à reconnaitre les mariages homosexuels en tant que tels, même s'ils ont été célébrés dans l'Etat de la résidence permanente de certains des requérants. Là encore, la Cour rappelle que

« there is also a State's legitimate interest in ensuring that its legislative prerogatives are respected and therefore that the choices of democratically elected governments do not go circumvented $» .55$

L'arrêt Orlandi (ou d'ailleurs l'arrêt, d'inspiration similaire, de la Cour de justice de l'Union européenne dans l'affaire $\operatorname{Coman}^{56}$ ) n'est assurément pas l'équivalent de l'arrêt Obergefell v. Hodges, ${ }^{57}$ un arrêt retentissant de la Cour suprême des Etats-Unis qui imposa à tous les Etats fédérés américains la reconnaissance du mariage entre personnes du même sexe. Encore faut-il observer que malgré les précautions, réelles, prises par la Cour européenne des droits de l'homme, une opinion dissidente des juges Aleš Pejchal (tchèque) et Krzysztof Wojtyczek (polonais) soulève des problèmes de principe à l'égard de la solution adoptée par la majorité, du point de vue de l'« effective political democracy $»$ :

« To sum up : in our view the majority have departed from the applicable rules of Convention interpretation and have imposed positive obligations which do not stem from this treaty. Such an adaptation of the Convention comes within the exclusive powers of the High Contracting Parties. We can only agree with the principle : "no social transformation without representation" ».58

\footnotetext{
53 Arrêt du 14 décembre 2017, nos 26431/12, 26742/12, 44057/12 et 60088/12.

54 Arrêt du 21 juillet 2017, Oliari et autres c. Italie, nos $18766 / 11$ et 36030/11.

55 Par. 207.

56 Arrêt du 5 juin 2018, Coman, C-673/16, ECLI:EU:C:2018:385. Pour un rapprochement des deux arrêts, cf. « European Courts and the Obligation (Partially) to Recognise Foreign Same-Sex Marriages - On Orlandi and Coman », Yb PIL, 2018/2019, 20, pp. 47 et seq.

57576 U.S. 644 (2015).

58 Par. 14 de l'opinion dissidente.
} 


\section{LE CONTRÔLE EUROPÉEN DE L'EMPLOI DE LA TECHNIQUE DE LA FRAUDE À LA LOI}

17. Aussi longtemps qu'elle persistait dans sa politique de refus de toute reconnaissance à la filiation entre des parents d'intention français et leurs enfants nés suite à une gestation pour autrui à l'étranger, la Cour de cassation française chercha, peut-être un peu désespérément, à rendre sa jurisprudence, attaquée par des parents d'intention déboutés, acceptable pour la Cour européenne des droits de l'homme. A un certain moment elle crut vraisemblablement avoir trouvé une manière plus pédagogique que le recours à l'ordre public international, en motivant le refus par le recours à la notion de fraude à la loi : « la naissance est l'aboutissement, en fraude à la loi française, d'un processus d'ensemble comportant une convention de gestation pour le compte d'autrui, convention qui, fût-elle licite à l'étranger, est nulle d'une nullité d'ordre public ».59

Mais ces efforts de renouvellement de la motivation du refus devaient s'avérer vains : dans l'affaire Foulon et Bouvet c. France, ${ }^{60}$ la Cour reprend les conclusions de ses arrêts Mennesson et Labassee (existence d'une violation de l'article 8 dans le chef des enfants pour méconnaissance de leur droit à l'identité, mais inexistence d'une violation de l'article 8 dans le chef des parents d'intention), et ceci de manière extrêmement brève : «Considérant les circonstances de l'espèce, la Cour ne voit aucune raison de conclure autrement que dans les affaires Mennesson et Labassee $» .^{61}$

Exit par conséquent la fraude à la loi comme justification d'une restriction au droit des enfants (qui n'auront certainement pas été parties ou complices à une opération frauduleuse aboutissant à leur propre naissance) à la reconnaissance de leur véritable filiation paternelle. Ceci ne signifie pas que jamais un motif de non-reconnaissance tiré d'une (véritable) fraude à la loi ne sera admis par la Cour européenne. Il n'y a aucune raison de le penser ; au contraire, si c'est par artifice qu'un futur requérant a acquis un statut à l'étranger qu'il n'aurait pas pu acquérir dans l'ordre juridique dans lequel il cherche pourtant à l'importer, le refus de cet ordre juridique de reconnaitre le statut acquis en fraude à la loi est évidemment susceptible d'être admis comme cause légitime d'ingérence dans la vie privée et familiale. Le fait que le requérant ait eu recours à des manœuvres frauduleuses lui interdit de faire état de la légitimité de sa confiance dans l'acquisition du statut à l'étranger, ce qui apparaît comme une précondition de la protection européenne du droit au respect de la vie familiale ou privée. ${ }^{62}$

59 Civ.1 1re, 13 septembre 2013, deux arrêts, no 12-18315 et no 12-30138. Cette jurisprudence a été abandonnée, par la suite, par Ass. plén., 3 juillet 2015, no 14-21323.

60 Arrêt du 21 juillet 2016, nos 9063/14 et 10410/14.

61 Par. 57.

62 Voir infra, no 20. 


\section{LE CONTRÔLE EUROPÉEN DU CHOIX MÉTHODOLOGIQUE ENTRE MÉTHODE DE LA RECONNAISSANCE ET MÉTHODE DE L'APPLICATION DE LA RÈGLE DE CONFLIT DU FOR À DES SITUATIONS CONSTITUÉES EN DEHORS DU FOR}

18. Comme le savent les spécialistes du droit international privé, une méthode générale de traitement des situations potentiellement boiteuses en droit international privé contemporain est la « méthode de la reconnaissance » qui propose de distinguer entre la création des situations dans un Etat, pour laquelle les règles de conflit de lois continueraient à s'appliquer, et le traitement des situations valablement acquises au regard d'un ordre juridique étranger, qu'il est proposé de reconnaître sans contrôle de la loi appliquée à l'étranger par rapport aux règles de conflit du for. ${ }^{63}$

Le droit international privé peut, en adoptant une variante ou une autre de la méthode de la reconnaissance, résoudre de manière autonome le problème des situations boiteuses résultant de la divergence des règles de conflit de lois ; un exemple en est l'article 9 de la Convention de La Haye du 14 mars 1978 sur la célébration et la reconnaissance de la validité des mariages qui impose, pour les (trois) Etats l'ayant ratifiée, la reconnaissance des mariages conclus à l'étranger, y compris par des ressortissants des Etats en cause, sans contrôle de la loi appliquée et sous réserve, pour l'essentiel, du seul ordre public. Il se peut aussi que si l'Etat du for (de la reconnaissance d'une situation constituée à l'étranger) reprend à son compte des solutions qui relèvent de la méthode de la reconnaissance, il le fasse uniquement parce qu'il est lié par l'un des textes ayant une portée quasi-constitutionnelle que sont le traité sur le fonctionnement de l'Union européenne d'une part, la Convention européenne des droits de l'homme d'autre part.

Même en matière de droit de la famille, la jurisprudence de la Cour de justice de l'Union européenne semble adhérer à une espèce de projet néolibéral dans le domaine des droits de la personne ou de la famille, très favorable à l'importation en cette matière de solutions se rattachant à la méthode de la reconnaissance ${ }^{64}: c^{\prime}$ est que pour elle, les liens de ce projet avec l'idée de libre circulation des personnes - centrale dans le droit de l'Union - sont évidents. C'est beaucoup plus discutable en revanche en ce qui concerne la Cour européenne des droits de l'homme.

19. Certes, la Cour européenne des droits de l'homme a eu l'occasion d'étendre et, en fait, d'universaliser la solution de deux arrêts de la Cour de justice auxquelles elle se réfère expressément, la jurisprudence Garcia Avello et Grunkin et Paul. ${ }^{65}$ Dans l'affaire Henry Kismoun c. France ${ }^{66}$ elle a imposé la possibilité pour un ressortissant de double nationalité algérienne et française de pouvoir faire valoir son intérêt à porter le même

${ }^{63}$ LAGARDE, P. « La méthode de la reconnaissance est-elle l'avenir du droit international privé ? », conférence publiée au Recueil des cours. 2014, tome 371, pp. 9 et seq. (avec une référence, p. 27, à la jurisprudence de la Cour européenne des droits de l'homme).

${ }^{64}$ Les décisions de principe sont connues : arrêts Garcia Avello du 2 octobre 2003, C-148/02, ECLI:EU:C:2003:539 ; Grunkin et Paul du 14 octobre 2008, C-353/06, ECLI:EU:C:2008:559 ; et des arrêts plus récents qui admettent cependant la justification de l'ingérence étatique à travers l'ordre public : Sayn-Wittgenstein du 22 décembre 2010, C-208/09, ECLI:EU:C:2010:806 ; Bogendorff von Wolffersdorff du 2 juin 2016, C-438/14, ECLI:EU:C:2016:401.

65 Les références de ces arrêts sont données supra, note précédente.

66 Arrêt du 5 décembre 2013, nº 32265/10. 
nom dans les deux Etats dont il était ressortissant et dès lors de pouvoir choisir un nom conforme au droit algérien, y compris pour le porter en France. Mais c'est par référence au droit du requérant à la vie privée et familiale (article 8 de la Convention), qui inclut un droit à l'identité dont un droit au nom, que la Cour européenne a décidé ainsi. Avec le changement de base juridique, cette jurisprudence change largement de sens par rapport à la jurisprudence de la Cour de justice de l'Union européenne. ${ }^{67}$

20. Dans la jurisprudence de la Cour européenne des droits de l'homme, l'obligation de reconnaître le statut juridique obtenu à l'étranger se fonde sur la protection de la confiance légitime des requérants. ${ }^{68} \mathrm{La}$ jurisprudence de la Cour est une jurisprudence inspirée d'un esprit d'équilibre et de compromis : elle ne reconnaît pas aux Etats la possibilité d'opposer, dans tous les cas, les conditions restrictives prévues par leur droit international privé à l'égard des situations constituées à l'étranger, mais elle ne proclame pas non plus la possibilité, sans restriction, pour les particuliers d'obtenir à l'étranger un statut juridique qui leur est refusé par le droit de l'Etat de leur nationalité ou de leur résidence, et de le faire reconnaître ensuite dans ce dernier Etat. Même si par ailleurs les relations entre les requérants peuvent se voir reconnaitre le statut d'une « vie familiale » de facto, la reconnaissance spécifique du statut juridique obtenu à l'étranger présuppose que ce statut ait été acquis de bonne foi et dans une situation de confidence légitime dans sa pérennité.

L'arrêt Wagner et J.M.W.L. c. Luxembourg69 - intervenu dès 2007 - a ainsi constaté l'existence d'une obligation pour le Luxembourg de reconnaitre l'adoption plénière de la requérante J.M.W.L. par $\mathrm{M}^{\mathrm{me}}$ Wagner au regard des circonstances de fait qui avaient entouré la procédure d'adoption au Pérou : $\mathrm{M}^{\mathrm{me}}$ Wagner s'était fiée à la pratique, alors existante, des officiers d'état civil luxembourgeois d'accepter la transcription automatique des jugements d'adoption étrangers, sans contrôle par rapport aux conditions formulées par le droit international privé luxembourgeois. Ayant adopté J.M.W.L., puis l'ayant emmenée au Luxembourg, elle s'était heurtée à une modification soudaine de la pratique administrative. Pourtant, comme le constate la Cour : « Une fois au Luxembourg, les requérantes pouvaient légitimement s'attendre à ce que l'officier d'état civil procédât à la transcription du jugement péruvien ». ${ }^{70}$

67 Non seulement la solution de l'arrêt Henry Kismoun intervient-elle à partir de bases conceptuelles très différentes (droit individuel au respect de l'identité personnelle plutôt que droit à la libre circulation des personnes dans l'Union européenne), mais encore la solution se trouve-t-elle ici universalisée, puisqu'elle s'applique dans les relations entre la France et l'Algérie et non pas dans le seul cadre, régional ou " fédéral », de l'Union. L'arrêt Henry Kismoun de la Cour européenne paraît également moins artificiel que certains aspects de la jurisprudence de la Cour de justice en matière de droit au nom : étant donné les limitations matérielles du champ d'application du droit de l'Union européenne, la Cour de justice est tenue, si elle veut intervenir dans ce domaine, de rattacher cette question à la libre circulation - ce qui est plausible dans l'affaire Sayn Wittgenstein (supra, note 63), mais beaucoup plus artificiel lorsqu'il s'agit de noms d'enfants, voire de bébés comme dans l'affaire Grunkin et Paul (ibid.).

68 Cf. «L'apport de la jurisprudence de la Cour européenne des droits de l'homme ». Dans LAGARDE, P. (dir.). La reconnaissance des situations en droit international privé. Paris : Pedone, 2013, pp. 43 et seq. ; «L'article 8 de la Convention et l'obligation de reconnaître les situations familiales constituées à l'étranger: à la recherche du fondement d'une solution jurisprudentielle ". Dans: Mélanges en l'honneur de Dean Spielmann. Oisterwijk : Wolf Legal Publishers, 2015, pp. 273 et seq.

69 Arrêt du 28 juin 2007, $\mathrm{n}^{\circ} 76240 / 01$.

70 Par. 130 de l'arrêt. 
De même, dans l'affaire Negrepontis-Giannisis c. Grèce, ${ }^{71}$ la non-reconnaissance en Grèce de l'adoption du requérant prononcée par un tribunal américain était de nature à porter atteinte à la confiance légitime du requérant, le fils adoptif, dans la validité de l'adoption. Le requérant avait été adopté par son oncle, un moine grec et évêque de l'Eglise orthodoxe à Detroit, dans des circonstances qui faisaient ressortir le caractère réel et substantiel des liens entre cette adoption et les Etats-Unis d'Amérique : le père adoptif était de nationalité grecque mais résident permanent aux Etats-Unis - de même que son neveu majeur résidait aux Etats-Unis depuis trois ans pour ses études universitaires. Ce ne fut qu'à la fin de ses études que le neveu et fils adoptif rentra en Grèce ; la relation de filiation adoptive fut maintenue pendant 14 ans, jusqu'au décès de l'oncle. On peut certes admettre que l'oncle devait être conscient du fait que l'adoption lui était interdite par le droit de l'Eglise orthodoxe grecque, mais il n'y a pas de raison de penser que son neveu aurait dû savoir qu'une vingtaine d'années plus tard, la Cour de cassation grecque allait décider, dans un litige sur la succession de l'oncle, que cette interdiction du droit ecclésiastique valait également pour le droit civil et faisait partie de l'ordre public au sens du droit international privé grec. ${ }^{72}$

Dans la décision, brièvement motivée, Green et Farhat c. Malte, ${ }^{73}$ la Cour était saisie d'une situation dans laquelle une citoyenne maltaise, qui avait épousé son premier mari à Malte conformément au rite catholique, s'expatria en Libye, s'y convertit à l'Islam (ce qui entraînait automatiquement, au regard de la loi libyenne, la dissolution de son mariage, une convertie ne pouvant rester liée à un non-musulman) et épousa, avec l'autorisation d'une juridiction locale, son deuxième mari, avec lequel elle resta pendant près de vingt ans en Libye où les époux étaient considérés comme valablement mariés. Vingt ans après, lors de son retour à Malte accompagné de son deuxième mari, elle échoua à obtenir l'enregistrement de son mariage libyen à Malte : les juridictions maltaises décidèrent qu'il n'était pas prouvé que le premier mariage de $\mathrm{M}^{\mathrm{me}}$ Green était réellement dissous et qu'en conséquence, le deuxième mariage semblait polygamique. La décision de la Cour rejette la requête comme manifestement malfondée : la prise en considération, par les juridictions maltaises, des intérêts de la communauté nationale au maintien du principe de la monogamie et de ceux du premier mari de la requérante restait à l'intérieur de la marge d'appréciation des autorités nationales. Les circonstances de fait de l'affaire Green et Farhat c. Malte intriguent, mais la décision est trop sommairement motivée pour permettre de dire si $\mathrm{M}^{\mathrm{me}}$ Green et son mari libyen étaient conscients, au moment de la conclusion de leur mariage, de ce que celui-ci ne serait pas reconnu à Malte, ni permet-elle de juger si la cour aurait été disposée à admettre que la très longue durée de la vie familiale en Libye était de nature à engendrer, à elle seule, une confiance légitime dans le chef de la requérante et de son second mari. En tout cas, il a été jugé qu'à supposer même qu'il y a ait eu ingérence dans la vie privée et familiale

71 Arrêt du 3 mai 2011, no 56759/08 (supra, no 12).

72 Par. 75 de l'arrêt : « la Cour relève que l'adoption litigieuse a eu lieu en 1984, alors que le requérant avait atteint l'âge adulte et qu'elle a duré vingt-quatre ans avant que la Cour de cassation n'y mette un terme par ses arrêts. Les parties n'ont par ailleurs fourni aucun élément tendant à démontrer que la réalité des liens entre le requérant et son père adoptif ait été mise en cause avant que la question de la succession ne se pose $"$.

73 Décision du 6 juillet 2010, no 38797/07. 
des requérants, les raisons d'ordre public invoquées par les autorités maltaises étaient suffisamment graves pour relever de la marge nationale d'appréciation.

21. Contrairement à l'apparence, peuvent également citées dans le présent contexte les affaires de non-reconnaissance en France de filiations issues de gestations pour autrui. ${ }^{74}$ La Cour y était confrontée à une situation où, selon ses constatations, les parents d'intention ne pouvaient ignorer « qu'il y avait au moins un risque sérieux » de non-reconnaissance en France de la filiation qui résulterait de la mise en œuvre de la convention de gestation pour autrui aux Etats-Unis. ${ }^{75} \mathrm{Ce}$ fait est de nature à montrer que la confiance légitime dans la reconnaissance de la filiation en France n'existait pas en ce qui concernait les parents d'intention. ${ }^{76}$ Toutefois, dans les arrêts de la Cour, une violation de la Convention ne fut constatée qu'à l'égard des enfants dont la filiation paternelle, conforme à la réalité biologique, n'était pas reconnue, violant ainsi leur droit à leur identité (article 8 de la Convention, au titre de la « vie privée ») : or les enfants nouveaux-nés n'avaient évidemment pas contribué aux circonstances de leur gestation, et la stabilité de leur situation familiale est pour eux une nécessité primaire, pouvant remplacer la « confiance légitime » qui ne peut pas exister dans leur chef.

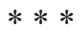

22. Pour terminer, voici une affaire portée devant la Cour européenne qui montre qu'occasionnellement, les buts poursuivis par la Convention européenne ne sont pas seulement compatibles avec les buts poursuivis par le droit international privé, mais y sont même identiques. ${ }^{77} \mathrm{Il}$ s'agit de l'affaire Chbihi Loudoudi et autres c. Belgique. ${ }^{78}$

Il s'agissait du rejet, par les tribunaux belges, d'une demande en adoption d'un enfant dont le statut personnel est celui d'un pays musulman (ici, le Maroc) qui prohibe l'adoption, enfant recueilli par kafala. C'est au moyen de règles matérielles de droit international privé que le droit international privé belge approche la question de l'adoption éventuelle d'un enfant confié en kafala. Ces règles ne se trouvant pas respectées en l'espèce, la demande en adoption de l'enfant, âgée de 14 ans et qui avait déclaré qu'elle souhaitait que l'adoption fût prononcée, ${ }^{79}$ a été rejetée.

Les requérants (les époux ayant recueilli l'enfant, ainsi que l'enfant lui-même) reprochent à la Belgique, devant la Cour européenne, d'avoir méconnu l'article 8 de la Convention (droit au respect de la vie familiale) en refusant, face à une vie familiale qui existe depuis la décision marocaine de kafala, d' « accorder une protection juridique

\footnotetext{
74 Voir supra, no 13.

75 Arrêt Mennesson c. France, par. 58, dans le contexte de la vérification du caractère « prévu par la loi » de l'ingérence.

76 Cf. en ce sens J. Guillaumé, note sous l'arrêt Mennesson au JDI 2014, p. 1273.

77 La même observation pouvait également être faite à propos d'un obiter dictum de l'arrêt Harroudj c. France (par. 51, cité supra, $\mathrm{n}^{\circ} 7$ ) consacré à une règle de conflit française : « en effaçant ainsi progressivement la prohibition de l'adoption, l'Etat défendeur, qui entend favoriser l'intégration d'enfants d'origine étrangère sans les couper immédiatement des règles de leur pays d'origine, respecte le pluralisme culturel et ménage un juste équilibre entre l'intérêt public et celui de la requérante ».

78 Arrêt du 16 décembre 2014, no 52265/10.

79 Par. 39.
} 
rendant possible l'intégration de l'enfant dans sa famille, ce qui ne serait le cas selon les requérants qu'au moyen d'une adoption ».

La Cour rejette leur requête. Elle retient d'abord que contrairement à la situation dans l'affaire Wagner et J.M.W.L. ${ }^{80}$ dont se prévalaient les requérants, ce n'est pas une adoption qui avait été prononcée à l'étranger, mais une kafala qui a certes créé un lien juridique entre les requérants «mais cette institution n'existant pas en Belgique, l'adoption qu'ils sollicitaient constituait, comme l'ont justement souligné les juridictions internes [...], une situation juridique nouvelle $\gg .81$

La Belgique n'a pas non plus méconnu son obligation positive de tout mettre en œuvre, dans l'intérêt supérieur de l'enfant, afin de protéger juridiquement la vie familiale entre les requérants. En effet, l'adoption n'est pas la seule procédure de protection des enfants abandonnés qui existe dans le monde, et la loi belge était destinée à mettre en œuvre l'objectif d'assurer que les adoptions internationales aient lieu dans l'intérêt (« supérieur ») de l'enfant à être protégé contre tout usage abusif de l'institution d'adoption et ainsi dans le respect de sa vie privée et familiale. Les tribunaux belges ne se sont pas abstenus d'une appréciation in concreto, mais ont jugé au contraire qu'il était conforme à l'intérêt bien compris de l'enfant de ne pas voir substituer en Belgique un autre statut (celui de l'adoption) au statut de la kafala seule reconnue au Maroc. Il convenait d'éviter que l'enfant ait en Belgique et au Maroc deux statuts personnels différents, celui d'un enfant adopté en Belgique et d'un enfant sous kafala au Maroc. La Cour juge que

« [1]es autorités belges pouvaient estimer ... que l'intérêt de l'enfant exigeait qu'elle n'ait qu'une et même filiation, aussi bien en Belgique qu'au Maroc (comparer, au sujet de l'importance pour une personne d'avoir un nom unique, Henry Kismoun c. France, no 32265/10, $\S 36,5$ décembre 2013)».82

Par conséquent, l'harmonie internationale des solutions apparaît ici comme une valeur importante, partagée par le droit international privé et la Convention européenne des droits de l'homme. La Cour européenne n'est pas l'ennemie du droit international privé ; à l'occasion, elle en est même l'alliée.

Professeur Patrick Kinsch, secrétaire général du GEDIP

Université du Luxembourg

patrick.kinsch@wka.lu

80 Supra, no 20.

81 Par. 91.

82 Par. 101. 